Part of Journal of Research of the National Bureau of Standards, Volume 18, June 1937

\title{
COMPENSATION OF STRAIN GAGES FOR VIBRATION AND IMPACT
}

\author{
By William M. Bleakney
}

\begin{abstract}
In general a strain gage, when attached to a vibrating member, undergoes deformations on account of the inertia of its parts, and these may cause serious errors in the strain readings. It has been customary in the past to decrease the mass and increase the rigidity of the gage in an effort to minimize these deformations and correct for the small resulting error. It is, however, frequently not feasible to construct a gage sufficiently light and rigid to make this possible. This paper describes methods for so adjusting the ratio of inertia to rigidity of the parts of the gage that these deformations are compensating. The indication of the instrument may thus be made independent of any acceleration of the gage as a whole.
\end{abstract}

\section{CONTENTS}

Page

I. Introduction

II. Statement of problem

III. Compensation principle

IV. Proposed application

\section{INTRODUCTION}

The demand for adequate instruments for the measurement of strain in structures in vibration or under impact loads is well known. In the study of vibration in aircraft the Bureau of Aeronautics of the U. S. Navy Department, in cooperation with the Massachusetts Institute of Technology, the Sperry Gyroscope Co., and the Engineering Mechanics Section of the National Bureau of Standards, has been interested in the development of such instruments which may be used to record vibratory strains in aircraft during flight. The severe vibration to which such instruments may be subjected in service requires unusual ruggedness combined with high sensitivity. The present paper is concerned, however, with another desirable attribute; namely, the faithful recording of strain independent of the amplitude and frequency of the vibrating structure under investigation.

\section{STATEMENT OF PROBLEM}

In general, strain gages consist of two or more parts, one part being attached to each of the two points $A$ and $B$ (fig. 1 and 2), between which the strain is to be determined. These two primary parts, here designated by $A^{\prime}$ and $B^{\prime}$, respectively, are restrained to 
relative motion of only one degree of freedom, which may be either translational (fig. 1) or rotational (fig. 2), and their relative displacement is indicated by a suitable measuring device $C$.

Figures 1 and 2 show diagrammatically two simple arrangements which can be used as strain gages. In practice the measuring device may take many different forms, such as a dial micrometer, an optical or mechanical lever, an electric resistance or impedance, an interferometer, or a microscope.

Such systems may be quite satisfactory for strain measurements under static conditions, but when they are applied to the measure-
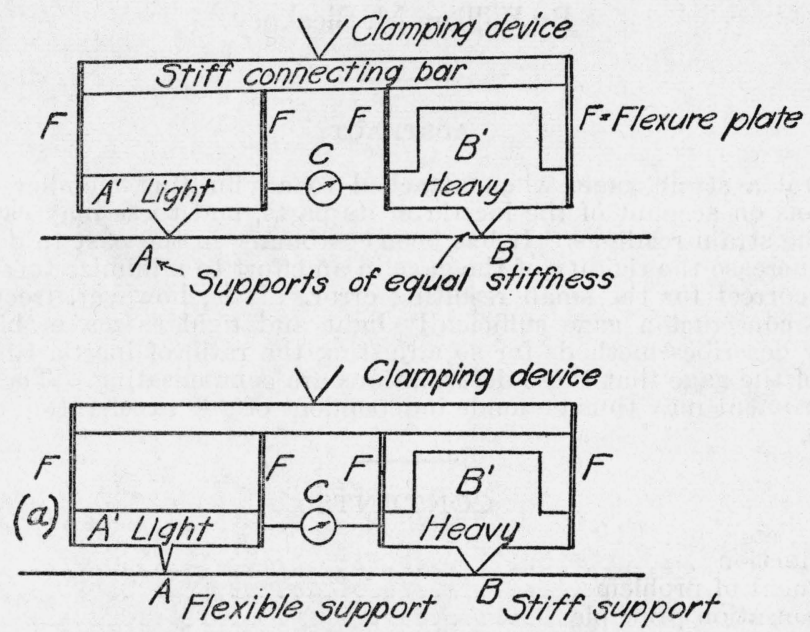

stiffness of supports proportional to masses

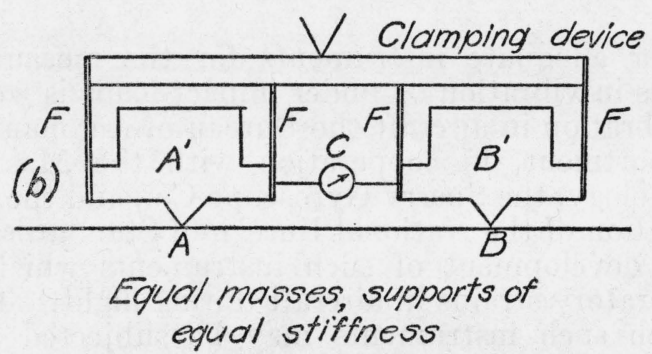

Figure 1.-Strain gage in which the relative motion of the primary parts $A^{\prime}$ and $B^{\prime}$ is translational.

ment of strains in structures in vibration or under impact loads, the accelerations of the systems produce inertia forces which may distort $A^{\prime}$ and $B^{\prime}$ and the connecting mechanism, so that the reading of $C$ is in general no longer definitely related to the relative motion of $A$ and $B$, but varies with the motion of the structure as a whole, and may also vary with the frequency of the vibratory motion.

To avoid this it has been customary in the past to make the parts of the strain-gage system as light and rigid as possible so that these deformations are minimized, and to correct for the small residual 
error by various methods, such as reversing the gage and taking the average of readings before and after reversal, ${ }^{1}$ or by control experiments to determine the effect of definite accelerations on the reading of the gage. These methods are only partially successful and become entirely inadequate in the study of very high frequency vibrations.

\section{COMPENSATION PRINCIPLE}

There is, however, another method, so far as we know not previously used, which extends the range of usefulness for these instruments. The method consists in so proportioning the stiffness to the inertia of parts of the system that the inertia forces alone produce no effect
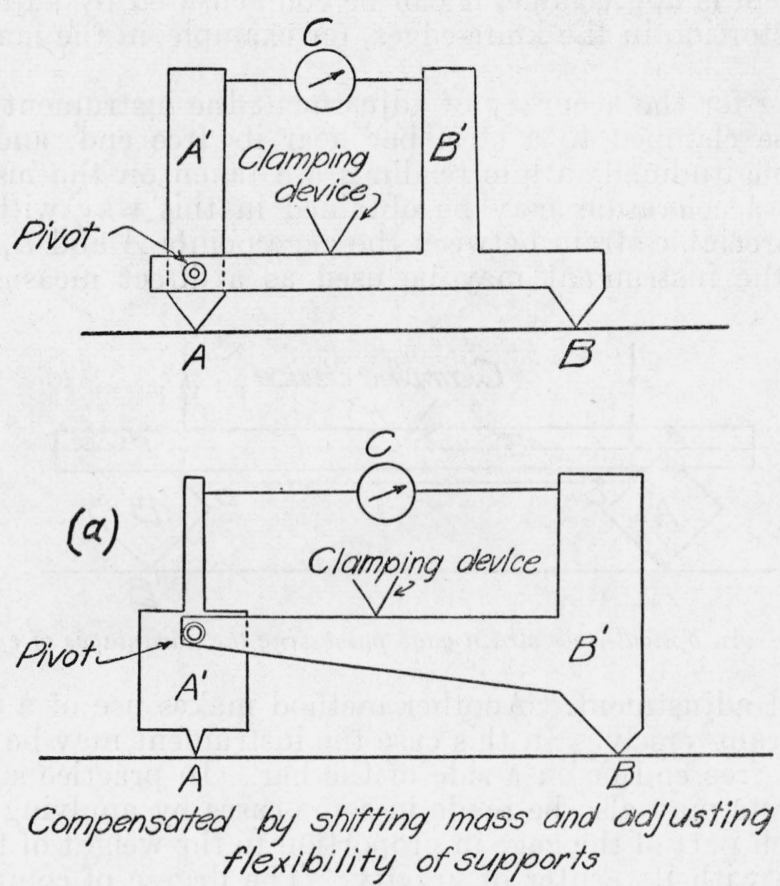

Figure 2.-Strain gage in which the relative motion of the primary parts $A^{\prime}$ and $B^{\prime}$ is rotational.

upon the indicating mechanism, although the individual parts of the gage are elastically deformed by these forces.

As a simple illustration of an application of this principle, consider the system represented in figure 1. If the system is accelerated along the line connecting $A$ and $B$, the knife-edges at these two points, for example, will become distorted and the instrument will in general indicate a strain, although no motion of $A$ relative to $B$ has occurred. If, however, the rigidity is modified as in figure 1 (a), or the mass distribution changed as in figure $1(\mathrm{~b})$, and final adjustment obtained by the removal of material at suitable points until no indication of strain is given under these conditions by the indicating mechanism $C$,

\footnotetext{
1 Ramberg, Ballif, and West. J. Research NBS 14, 189 (1935) RP764.
} 
the gage is then compensated for this type of acceleration, and, of the possible motions of $A$ and $B$ parallel to the line $A B$, only relative displacements will be indicated by the instrument.

As a second illustration, consider the system represented in figure 2 . This system may be modified as shown in figure 2 (a) to provide compensation for longitudinal acceleration of the gage as a whole. The center of mass of the rotating part is shifted below the pivot so that the inertia forces will produce reactions on the knife-edges, which are in the same direction. Distortions in the knife-edges may now be made to compensate by making these reactions proportional to the stiffness of the knife-edges, either by altering the stiffness, or by changing further the mass distribution. If distortion in other parts of the system is appreciable, it can be compensated by adjusting the relative distortion in the knife-edges, for example, in the manner just described.

In testing for the accuracy of adjustment the instrument may, for example, be clamped to a steel bar near its free end, and the bar vibrated longitudinally while readings are taken on the instrument. Since high acceleration may be obtained in this way without producing appreciable strain between the gage points $A$ and $B$, the indication of the instrument may be used as a direct measure of the

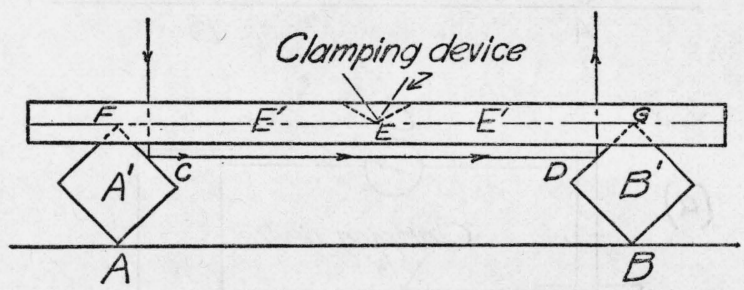

FIGURE 3.-An optical-lever strain gage possessing the advantages of symmetry.

accuracy of adjustment. Another method makes use of a thick bar vibrating transversely. In this case the instrument may be mounted either on a free end or on a side of the bar. In practice sufficiently accurate tests may also be made in some cases by applying a steady force to each part of the gage in proportion to the wejght of that part, and in line with its center of gravity. The degree of compensation for acceleration in the direction of these forces will then be indicated by the instrument, provided the principal deformations are localized so that the major portion of each part may be considered practically rigid.

Figure 3 illustrates a simple application of the compensation principle to an optical lever strain gage. The strain is indicated by the rotation of $A^{\prime}$ relative to $B^{\prime}$, this rotation being measured by reflecting a light beam from these two parts in succession. By making the system symmetrical about a vertical line through $E$, deformation arising from acceleration of the system parallel to the line $A B$ will be compensating. Thus extension in $G E$, for example, will be balanced by compression in $E F$, so that the length $G F$ remains unchanged. Deformation in the knife-edges of $A^{\prime}$ and $B^{\prime}$, should it occur, will be compensated in a similar manner.

The effect of vertical acceleration of the system upon the reading of the instrument may also be reduced and practically compensated 
by making the points $F$ and $G$ lie on the neutral axis in bending of the member $E^{\prime}$. Thus changes in the length of $F G$ are reduced to second order effects with respect to this type of motion. In this way the gage may be compensated for simultaneous effects arising from two different types of inertia forces.

Thus far we have considered inertia forces arising from acceleration of the strain gage as a whole. In general, these are the most troublesome, first because they may be many times larger than the inertia forces arising from the relative motion of the parts of the gage, and second they are not determined by the strain which is to be measured.

In some cases, however, it may be desirable to compensate a gage for distortions arising from the relative motion inherent in the strain itself, for these distortions may cause the reading of the instrument to depend upon the frequency as well as the amplitude of the vibrating strain. Figure 3 may be used again to show how the present method may be applied to reduce or eliminate this frequency effect.

In this system the rocking members $A^{\prime}$ and $B^{\prime}$ rotate about axes through $F$ and $G$, respectively, in response to strain in the line $A B$, and angular acceleration about these axes gives rise to the inertia forces at $A$ and $F$ and at $B$ and $G$, which are to be compensated. It may easily be shown, for instance, that if $A^{\prime}$ is square in cross section the forces acting due to this cause at $A$ and $F$ are in the same direction and in the ratio of $2: 1$. It remains only to adjust the rigidity of $E F$ so that the combined distortion of $E F$ and the knife-edge at $F$ balances the distortion of the knife-edge at $A$, thus preventing rotation of $A^{\prime}$ as a result of these deformations. When a similar adjustment is made for $B^{\prime}$ the frequency effect will be eliminated.

This adjustment will not necessarily affect the adjustment for acceleration of the gage as a whole in the plane of the drawing, as described above. Compensation may thus be obtained simultaneously for three distinct types of error.

\section{PROPOSED APPLICATION}

Figure 4 is a simplified drawing to illustrate the immediate application of this principle which is now contemplated. The relative longi-

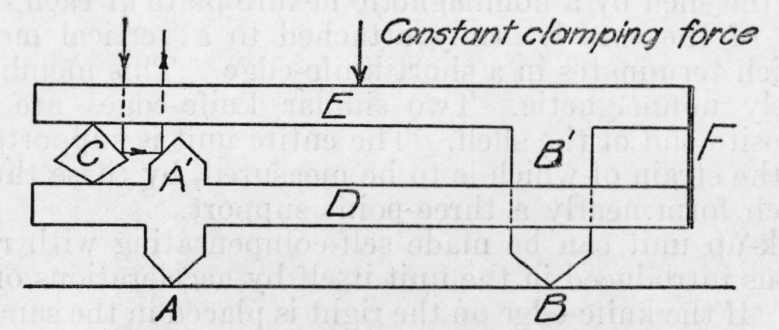

FiguRE 4.-Compensalion principle as applied in figure 5.

tudinal motion of the horizontal members $D$ and $E$ may be measured by any suitable indicating device. For simplicity an optical system is indicated. The flexure plate $F$ may be designed to carry compressive loads in order to stabilize the system for a constant clamping. force applied as indicated. Compensation for acceleration of the 
system parallel to the line $A B$ is accomplished in the same manner as that described above. Vertical accelerations will in general produce bending in the horizontal members $D$ and $E$, which will result in the tipping of the vertical parts $A^{\prime}$ and $B^{\prime}$. This may result in a spurious indication of the instrument. It is clear that by shifting the position of the rocker $C$ the relative bending moments acting on $D$ and $E$ may be so adjusted that the individual effects compensate each other. In practice, the member $D$, for instance, may be so rigid compared to $E$ that shifting the position of $C$ has little effect. Adjustment may then be made by choosing the proper distance between the point $B$ and the plane of the flexure plate.

This latter method is used in the proposed electromagnetic strain pick-up unit sketched in figure 5. The electrical features of the design were developed by the Massachusetts Institute of Technology and the Sperry Gyroscope Co., who are collaborating in this work. ${ }^{2}$

Figure 5 shows a longitudinal midsection view, which illustrates the essential features of the unit. The core is a permanently magnetized rod of high retentivity and coercivity. Two pairs of soft iron

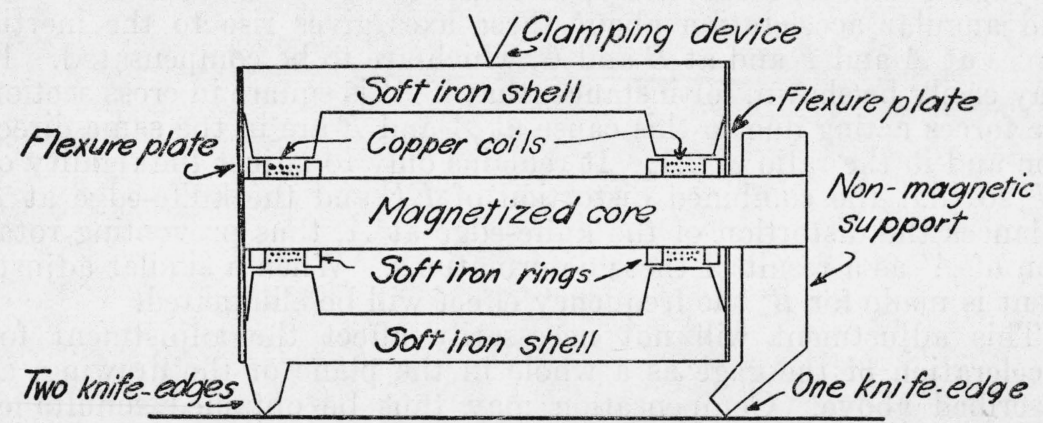

FIGURE 5.-Electromagnetic and mechanical features of the proposed strain pick-up unit.

rings are sweated on as shown, and insulated copper coils are wound between the rings of each pair. A cylindrical soft iron shell closes the magnetic circuit. The core is restrained to longitudinal motion relative to the shell by a nonmagnetic flexure plate at each end.

One end of the core is firmly attached to a vertical member, as shown, which terminates in a short knife-edge. This member should be relatively nonmagnetic. Two similar knife-edges are attached to the opposite end of the shell. The entire unit is supported on the structure, the strain of which is to be measured, by these three knifeedges, which form nearly a three-point support.

This pick-up unit can be made self-compensating with respect to deformations introduced in the unit itself by accelerations of the unit as a whole. If the knife-edge on the right is placed in the same vertical plane as the flexure plate at that end, the effect of vertical accelerations will be nearly eliminated. If flexure in the shell and core attributable to vertical accelerations, combined with vertical strain in the flexure plates, is still sufficient to cause appreciable error, this can be compensated by shifting the right knife-edge slightly with respect to

${ }^{2}$ Draper, Bentley, and Willis. J. Aeron. Sci. 4, 281 (1937). 
the flexure plate. The optimum position for this knife-edge may be found by trial and henceforth incorporated permanently in the unit.

More serious errors may arise from internal deformations because of longitudinal accelerations of the unit as a whole. These errors, however, may be completely compensated by making the ratio of mass to rigidity the same in the two members. This can be done by trial on each individual unit by removing material at suitable points until strain readings are shown by test to be independent of longitudinal acceleration. This adjustment may be simplified by making the knife-edges similar in shape, but with a length in proportion to the horizontal load they must carry. In making this balance the effects of inertia reactions of the clamping device on the unit should be compensated.

It is desirable to make the unit as rigid as practicable in order to minimize errors caused by incomplete compensation with respect to translational accelerations as just described. For this reason blunt knife-edges are suggested. There remain possible errors caused by rotational acceleration of the unit as whole, and by the small differences in acceleration of the two members arising from the strain which is to be measured. However, the unit as designed is so rigid that these latter errors will not be appreciable in any application contemplated at present.

The methods outlined here for eliminating errors in the measurement of vibratory or transient strains when these errors arise from deformations within the gage itself have not, to our knowledge, been applied or described previously in the literature. It is to be expected that, as improved instruments for making such measurements become available, these methods will become increasingly important.

Washington, March 31, 1937. 